\title{
The OPUS Effect: An Investigation of how Magnum OPUS may Influence Athletic Performance
}

\author{
Nicholas Conley ${ }^{\mathrm{a}}$ and Jessica Campisi ${ }^{\mathrm{a}}$
}

The goal of this study was to investigate the Magnum OPUS supplement in terms of its ability to enhance athletic performance and possess a tolerable safety profile. Two groups (placebo and experimental) performed a series of exercises (pushups, dips, squats, lunges) with each repetition being recorded and compared to the baseline. Upon completion of four sessions by all twenty participants, the results were scored and significant differences were noted $(\mathrm{t}(18)=-5.41, \mathrm{p}<0.001,95 \%$ CI $[-24.25,-10.68])$. In addition, participants recorded all adverse effects experienced with the use of the supplement. It was concluded that the participants taking OPUS had a significant increase in athletic performance when compared against the placebo group. OPUS was shown to be a reasonably safe supplement that may help athletes with their training and other athletic activities.

Keywords: athletic supplement, athletic performance, beta alanine

\section{Introduction}

There are hundreds of pre-workout supplements on the market that claim to help athletes improve performance on some measurable scale. By visiting a local store or performing a quick Internet search, one can see the multitude of products available. These different products claim to do everything from helping the user gain muscle, lose weight, recover faster, get stronger, and improve their overall wellbeing ${ }^{19}$.

While these supplements are widely available, the Food and Drug Administration (FDA) do not regulate them. Therefore, most of these nutritional products do not undergo strict precautions, guidelines, and testing to evaluate the claims being made. This often results in disappointed consumers and a scarcity of supplements providing the benefits they have claimed ${ }^{31}$. In turn, the consumer spends increasing amounts of money trying to not only find the right product, but also finding one that is safe and effective to use.

In today's supplement industry, companies are able to put an unsafe and unproven product on the market in order to avoid the extensive time and research to scientifically test their products through clinical studies. This can lead to deceptive labeling, outlandish promises, and proprietary blends to help mask the supplement's ingredients from the consumers ${ }^{19}$. By avoiding this extra time and cost, companies are able to increase revenue and sales. However, the average consumer has no idea which companies they can trust and may potentially prey to a deceptive market ${ }^{19}$. This has created a high level of skepticism about the supplement industry and supplements themselves are often considered fake or harmful. This is not true in all cases, however the majority of the companies making these supplements do not have enough research and evidence to support the use of their products.

Magnum Nutraceuticals' OPUS was designed and developed to help athletes push further in training and exercise thus allowing them to increase strength, agility, and endurance. OPUS contains CarnoSyn ${ }^{\circledR}$ beta alanine an essential amino acid, which works by synthesizing carnosine and acting as a precursor to nitric oxide. Carnosine functions as a buffer to maintain optimal muscular $\mathrm{pH}$ while nitric oxide is a vasodilator increasing blood flow to skeletal muscle ${ }^{22}$. In addition to blood flow, the skeletal muscle is also supplied with an increase of nutrients and oxygen. These properties encourage a favorable environment that supports muscle function and growth. While having these effects, beta alanine has shown few side effects and maintains a tolerable safety profile. Because beta alanine is an amino acid, and normally found in the human body, it is safe for use in normal amounts. The only adverse effects known to date is that of flushing and paresthesia, which is a result of the vasodilator properties of the supplement. This side effect occurred more commonly when beta alanine was given in high doses. This adverse effect, however, disappeared within 1 hour after ingestion ${ }^{1}$ and appears self limiting. A full list of active ingredients can be seen in Figure 1. This study will experiment with OPUS in a variety of athletes in an effort to provide evidence on how much it can really improve athletic performance. The null hypothesis is that the intervention group will have an increate of athletic performance when compared to the placebo group.

\section{Methods}

Participants

There were twenty participants, both male and female, recruited for this study. Each participant was over the age of 18 and either currently attending Wingate University or from the surrounding area of Wingate, North Carolina. The participants were obtained from a series of school emails that were sent to the student body seeking those who regularly competed in athletics to volunteer for this study. To be considered, selected participants had to be involved in at least two sports. Exclusion criteria involved any history of significant injury or medical issue that would prevent them from taking part in the selected exercises. 


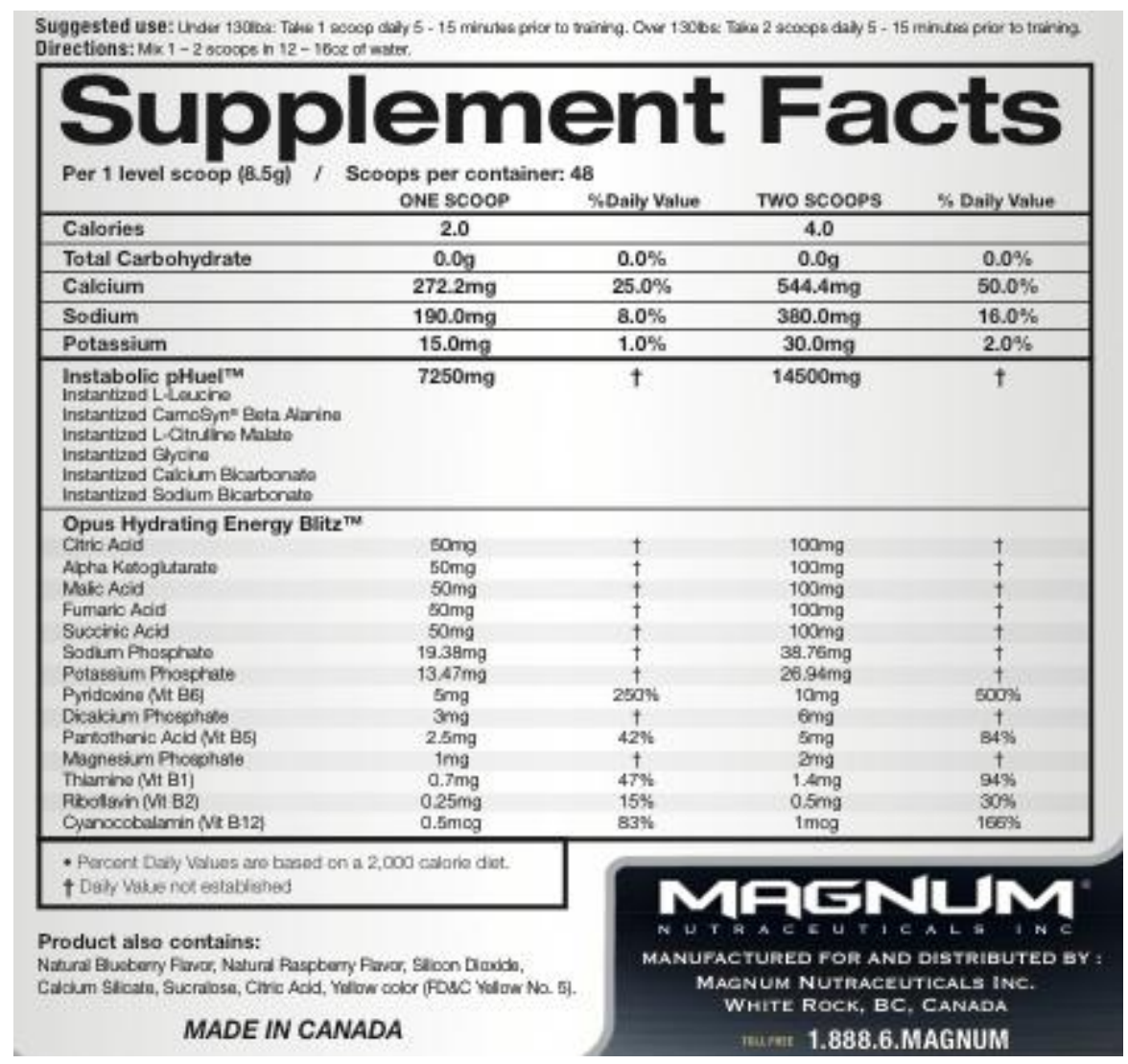

Figure 1. Supplemental label of Magnum OPUS

The participants were informed that this research project was being used to collect data and information on the supplement OPUS and how it could influence athletic performance. All personal information regarding the participants will be kept confidential and will not be released to the public.

\section{Design}

This study used a between subject experimental design that was also double-blinded. The independent variable consisted of the participant receiving the intervention (OPUS) or the placebo. The dependent variable was the average increase of repetition performed during each session. Overall increase in athletic performance was defined as the percentage change from baseline when all repetitions from each type of exercise were averaged for each participant. The placebo group $(\mathrm{N}=10)$ combined with the OPUS group $(\mathrm{N}=10)$ gave a total number of 20 participants involved (Figure 2). The Wingate University research review board approved this study.

\section{Materials}

The supplement OPUS and the Placebo were both provided by Magnum Nutraceuticals. When OPUS reacts with water, it produces an effervescent effect, which turns the water into a green solution releasing the active ingredients. Provision of OPUS 15 minutes before activity was chosen according to recommendation by the manufacturer. The placebo was constructed with these same properties as to react substantially similar to the intervention and protect the integrity of the study. A brief survey was also developed by the researcher to help collect the opinions of the participants in regards to OPUS as well as to assist with the collection of exercise repetitions (Figure 3). The survey used an ordinal scale in which participants ranked the supplement based on safety and efficacy. The mean age of the participants involved was 23.55 years old. The placebo group had an average age of 23.6 years old while the intervention group had an average age of 23.50 years old.

\section{Procedures}

After informed consent was received from all twenty participants, they were categorized into groups using simple randomization. All participants were instructed that they would individually meet with a researcher during four exercise sessions that were separated by seven days of rest. The participants were asked to resume any daily activity, as they normally would while taking part in the study. There were four different exercises using the participants' own body weight (pushups, dips, squats, and lunges) and they were given five minutes per each exercise to complete as many repetitions as they could. There was a thirty-second rest period between each exercise. These activities and lengths of duration were chosen due to the proposed mechanism of beta alanine. 


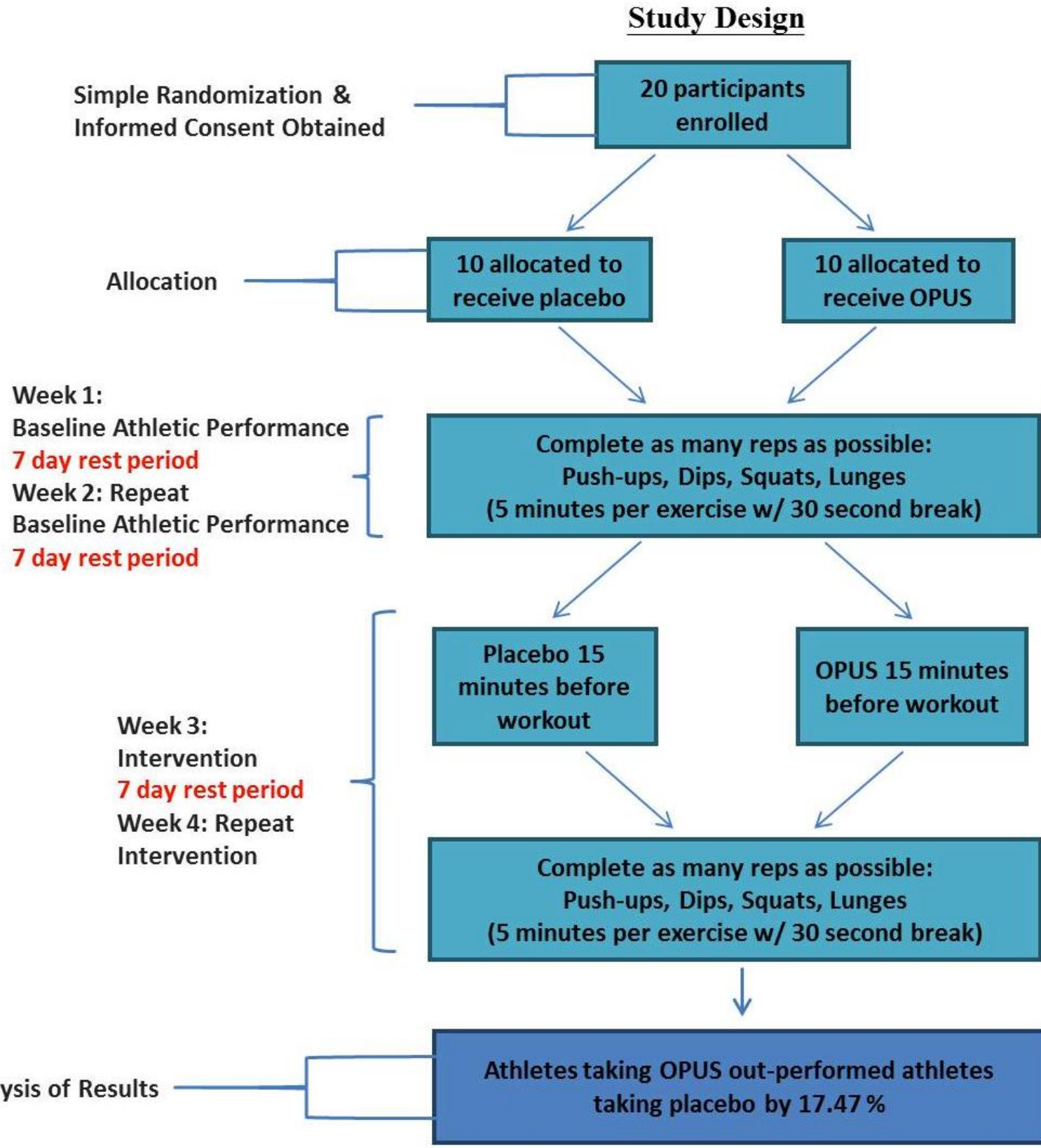

Figure 2. Patient allocation, procedure, and time to analysis of $O P U S$ trial

The greatest effects of increased buffering capacity are observed in activities that produce a high amount of lactic acidosis. This is seen in activities lasting from 60 second to 5 minutes especially during exercises that work the same muscle groups and induce fatigue ${ }^{1}$. In addition, these activities were chosen based upon their lack of necessary training or advanced level of athleticism to perform them. All repetitions were recorded. A 30 second break was provided to allow participants to rest without having time to completely recover $^{25}$. The first two sessions served as the participant's baseline and for the final two sessions the participant was introduced to the interventions.

The reason these four particular exercises were selected was because pushups with dips and squats with lunges all work similar muscle groups. If these exercises are repeated as they were during the study then it is likely that the participant experienced a burnout period, meaning the muscle groups would be exhausted, thus resulting in a failed repetition. This would help measure the claim of OPUS as it promises to increase athletic performance through the delay of muscle fatigue.

During the final two sessions, the ten participants in the OPUS group were given two scoops of the supplement mixed with $12 \mathrm{oz}$ of water as instructed by the manufacturer. After waiting 15-minutes (allowing time for the supplement to be systemically absorbed as claimed by the manufacturer), the participant was then asked to complete the four exercises as they have previously done the prior weeks. This set of data 
was collected and compared with their baseline numbers. During the final two sessions, the ten participants in the Placebo group were given two scoops of the Placebo mixed with $12 \mathrm{oz}$ of water. These participants also had a 15-minute wait time before engaging in the four exercises.

Four-trained doctorate students from Wingate University School of Pharmacy conducted the individual sessions of the participants. Each trained student acted as a research assistant and individually led a group of five participants. This utilized a double blind design because the lead investigator was now able to blind each assistant while they administered either the placebo or OPUS to the participants.

At the conclusion of the final session, participants were asked to answer a survey that collected their opinions of OPUS. Each participant answered the survey under the impression that they had received the supplement. However, only the data collected from participants that had actually received the supplement (not placebo) were used. A fivepoint scale was used with five being the highest agreeability and one being the lowest. The questions were as follows: 1-I believe that OPUS has improved my overall athletic ability; 2-I would recommend OPUS to other athletes; 3 -I believe that OPUS improved my overall energy level; and 4-I felt OPUS was reasonably safe to use. In addition to the survey responses, the participants were asked to list their opinion regarding OPUS as well as about the sports they were involved in.

Statistical analysis

All data was collected, measured and scored by the student researchers involved. An independent t-test was used to conclude if there was a difference between primary endpoint of the OPUS group and the placebo group. The primary end point was an average change of all activities therefore only two variables were used. An alpha of 0.05 was set to determine significance between the two independent continuous variables.

\section{Results}

Statistical analysis was performed to conclude if the increase in athletic performance recorded was due to the supplement OPUS, $\mathrm{t}(18)=-5.41, \mathrm{p}<0.001,95 \%$ CI $[-24.25,-10.68]$. The data was concluded statistically significant and thus the null hypothesis was rejected. All participants that took OPUS experienced an increase in athletic performance. The highest was participant \#1 who had an increase of $32.95 \%$ and the lowest was participant \#15 with $4.97 \%$. These percentages show the change when all repetitions from each exercise were averaged at both baseline and at intervention. The average increase of athletic performance was $17.60 \%$ for participants taking OPUS. The participants that took the Placebo had only an average increase of $0.13 \%$. The highest performance experienced was $10.26 \%$ by participant \#8 while the lowest was a decrease in athletic performance by participant \#10 of $12.46 \%$. Those that took OPUS outperformed the Placebo group by $17.47 \%$. The standard deviation for the Placebo group was 6.23. The standard deviation for the OPUS group was 8.08. Additional differences between groups and for each particular type of exercise can be seen in Table 1 . The opinion survey was completed on the final session and was averaged across participants that received OPUS: I felt OPUS was reasonably safe to use (4.8/5); I felt OPUS increased my overall energy levels (4/5); I would recommend OPUS to other athletes (3.4/5); and I felt OPUS improved my overall athletic ability (3.6/5). From the ten participants that took OPUS the following adverse reactions were reported: Pruritus (4/10), Dyspepsia (3/10), Eructation (2/10), and None (2/10). Pruritis was likely caused by the beta alanine, as skin sensations are the most commonly reported adverse effect. Dyspepsia and eructation were reported in some of the participants taking OPUS and were potentially due to the effervescent properties of the supplement. When comparing beta alanine to other popular supplements on the market, the incidence of serious adverse effects is much smaller. For example, creatinine supplementation has reports of muscle cramping $(25 \%)$, gastrointestinal pain, nausea, diarrhea, weight gain, and rare incidences of renal dysfunction while dimethylamylamine supplementation has serious risks of cardiovascular events ${ }^{22}$. While OPUS did have reports of adverse effects, no participants discontinued the trial due to these occurences.

\section{Discussion}

The purpose of this research project was to examine the claim from Magnum Nutraceuticals in regards to their new sports supplement OPUS. Magnum Nutraceuticals reports that this product will increase athletic performance and this study will test this notion in athletes during repetitive exercises.

Previous studies have shown that supplementation may have varying effects in individuals based on a number of characteristics or habits including ingestion timing, mode of ingestion, and type of activities performed ${ }^{13}$. Therefore, consumers may have to experiment with several manufacturers and products before identifying the one that works best for them ${ }^{19}$. While OPUS may not be the product for everyone, this study may suggest that the product could help increase athletic performance during repetitive exercises for the selected population.

This study has a number of limitations that are summarized below. Due to limited resources, the sample size only included twenty participants. Future research should be done with a larger sample size to see if results are duplicated. This research should also include a third arm (control, placebo, OPUS) trial. The data showed a difference in means when comparing the Placebo versus the OPUS group. This could be due to many factors including the lack of stratification among groups. There were no baseline characteristics describing the normal activity levels or supplement-use history of each of the participants. The results could be due to natural improvement in athletic performance as a result of exercise performed during weekly assessments as opposed to the actual benefit from the supplement in participants that have less experience with these types of activities.

This study provides statistically significant data suggesting that OPUS can help athletes increase athletic performance during repetitive exercises. With further research containing larger sample sizes, more conclusions can be made as to the clinical applications of this supplement. 
Table 1. Results from OPUS users including overall percent change from baseline and percent changes for each particular exercise

\begin{tabular}{|c|c|c|c|c|}
\hline Participant \# & Baseline averages & OPUS averages & $\%$ Change & Conclusion \\
\hline $\begin{array}{c}1 \\
\text { (25 y/o Male) }\end{array}$ & $\begin{array}{l}\text { Push-ups: } 88.5 \\
\text { Dips: } 72 \\
\text { Squats: } 133.5 \\
\text { Lunges: } 124.5\end{array}$ & $\begin{array}{l}\text { Push-ups: } 112.5 \\
\text { Dips: } 110.5 \\
\text { Squats: } 181 \\
\text { Lunges: } 144\end{array}$ & $\begin{array}{l}\text { Push-ups: } \uparrow 27.11 \% \\
\text { Dips: } \uparrow 53.47 \% \\
\text { Squats: } \uparrow 35.58 \% \\
\text { Lunges: } \uparrow 15.66 \%\end{array}$ & $\begin{array}{l}\text { OPUS increased } \\
\text { overall athletic } \\
\text { performance } \\
32.95 \%\end{array}$ \\
\hline $\begin{array}{c}2 \\
\text { (20 y/o Female) }\end{array}$ & $\begin{array}{l}\text { Push-ups: } 90 \\
\text { Dips: } 65.5 \\
\text { Squats: } 127.5 \\
\text { Lunges: } 104\end{array}$ & $\begin{array}{l}\text { Push-ups: } 95.5 \\
\text { Dips: } 111 \\
\text { Squats: } 168.5 \\
\text { Lunges: } 114.5\end{array}$ & $\begin{array}{l}\text { Push-ups: } \uparrow 6.11 \% \\
\text { Dips: } \uparrow 69.46 \% \\
\text { Squats: } \uparrow 32.15 \% \\
\text { Lunges: } \uparrow 10.09 \%\end{array}$ & $\begin{array}{l}\text { OPUS increased } \\
\text { overall athletic } \\
\text { performance } \\
29.45 \%\end{array}$ \\
\hline $\begin{array}{c}3 \\
\text { (22 y/o Female) }\end{array}$ & $\begin{array}{l}\text { Push-ups: } 108.5 \\
\text { Dips: } 116.5 \\
\text { Squats: } 197.5 \\
\text { Lunges: } 152.5\end{array}$ & $\begin{array}{l}\text { Push-ups: } 122 \\
\text { Dips: } 150 \\
\text { Squats: } 216 \\
\text { Lunges: } 158\end{array}$ & $\begin{array}{l}\text { Push-ups: } \uparrow 12.44 \% \\
\text { Dips: } \uparrow 28.75 \% \\
\text { Squats: } \uparrow 9.36 \% \\
\text { Lunges: } \uparrow 3.60 \%\end{array}$ & $\begin{array}{c}\text { OPUS } \underline{\text { increased }} \\
\text { overall athletic } \\
\text { performance } \\
13.53 \%\end{array}$ \\
\hline (25 y/o Male) & $\begin{array}{l}\text { Push-ups: } 48 \\
\text { Dips: } 53 \\
\text { Squats: } 91.5 \\
\text { Lunges: } 118\end{array}$ & $\begin{array}{l}\text { Push-ups: } 49.5 \\
\text { Dips: } 62.5 \\
\text { Squats: } 116.6 \\
\text { Lunges: } 121\end{array}$ & $\begin{array}{l}\text { Push-ups: } \uparrow 3.12 \% \\
\text { Dips: } \uparrow 17.92 \% \\
\text { Squats: } \uparrow 27.32 \% \\
\text { Lunges: } \uparrow 2.54 \%\end{array}$ & $\begin{array}{c}\text { OPUS increased } \\
\text { overall athletic } \\
\text { performance } \\
12.72 \%\end{array}$ \\
\hline $\begin{array}{c}5 \\
\text { (25 y/o Male) }\end{array}$ & $\begin{array}{l}\text { Push-ups: } 65.5 \\
\text { Dips: } 75.5 \\
\text { Squats: } 105.5 \\
\text { Lunges: } 87\end{array}$ & $\begin{array}{l}\text { Push-ups: } 69.5 \\
\text { Dips: } 90 \\
\text { Squats: } 125.5 \\
\text { Lunges: } 100\end{array}$ & $\begin{array}{l}\text { Push-ups: } \uparrow 6.10 \% \\
\text { Dips: } \uparrow 19.20 \% \\
\text { Squats: } \uparrow 18.95 \% \\
\text { Lunges: } \uparrow 14.94 \%\end{array}$ & $\begin{array}{c}\text { OPUS increased } \\
\text { overall athletic } \\
\text { performance } \\
14.79 \%\end{array}$ \\
\hline $\begin{array}{c}11 \\
\text { (22 y/o Male) }\end{array}$ & $\begin{array}{l}\text { Push-ups: } 91 \\
\text { Dips: } 72.5 \\
\text { Squats: } 140.5 \\
\text { Lunges: } 126.5\end{array}$ & $\begin{array}{l}\text { Push-ups: } 96.5 \\
\text { Dips: } 104 \\
\text { Squats: } 167 \\
\text { Lunges: } 120\end{array}$ & $\begin{array}{l}\text { Push-ups: } \uparrow 6.04 \% \\
\text { Dips: } \uparrow 43.44 \% \\
\text { Squats: } \uparrow 18.86 \% \\
\text { Lunges: } \downarrow 5.13 \%\end{array}$ & $\begin{array}{c}\text { OPUS increased } \\
\text { overall athletic } \\
\text { performance } \\
15.80 \%\end{array}$ \\
\hline $\begin{array}{c}12 \\
\text { (21 y/o Male) }\end{array}$ & $\begin{array}{l}\text { Push-ups: } 130 \\
\text { Dips: } 107 \\
\text { Squats: } 113 \\
\text { Lunges: } 103\end{array}$ & $\begin{array}{l}\text { Push-ups: } 140 \\
\text { Dips: } 137.5 \\
\text { Squats: } 141.5 \\
\text { Lunges: } 107.5\end{array}$ & $\begin{array}{l}\text { Push-ups: } \uparrow 7.69 \% \\
\text { Dips: } \uparrow 28.50 \% \\
\text { Squats: } \uparrow 25.22 \% \\
\text { Lunges: } \uparrow 4.36 \%\end{array}$ & $\begin{array}{l}\text { OPUS increased } \\
\text { overall athletic } \\
\text { performance } \\
16.44 \%\end{array}$ \\
\hline $\begin{array}{c}13 \\
\text { (24 y/o Male) }\end{array}$ & $\begin{array}{l}\text { Push-ups: } 117.5 \\
\text { Dips: } 83 \\
\text { Squats: } 74.5 \\
\text { Lunges: } 53.5\end{array}$ & $\begin{array}{l}\text { Push-ups: } 127.5 \\
\text { Dips: } 94 \\
\text { Squats: } 102.5 \\
\text { Lunges: } 60.5\end{array}$ & $\begin{array}{l}\text { Push-ups: } \uparrow 8.51 \% \\
\text { Dips: } \uparrow 13.25 \% \\
\text { Squats: } \uparrow 37.58 \% \\
\text { Lunges: } \uparrow 13.08 \%\end{array}$ & $\begin{array}{l}\text { OPUS increased } \\
\text { overall athletic } \\
\text { performance } \\
18.10 \%\end{array}$ \\
\hline $\begin{array}{c}14 \\
\text { (25 y/o Male) }\end{array}$ & $\begin{array}{l}\text { Push-ups: } 122.5 \\
\text { Dips: } 71.5 \\
\text { Squats: } 76 \\
\text { Lunges: } 63.5\end{array}$ & $\begin{array}{l}\text { Push-ups: } 137.5 \\
\text { Dips: } 81.5 \\
\text { Squats: } 92.5 \\
\text { Lunges: } 77\end{array}$ & $\begin{array}{l}\text { Push-ups: } \uparrow 12.24 \% \\
\text { Dips: } \uparrow 13.98 \% \\
\text { Squats: } \uparrow 21.71 \% \\
\text { Lunges: } \uparrow 21.25 \%\end{array}$ & $\begin{array}{c}\text { OPUS increased } \\
\text { overall athletic } \\
\text { performance } \\
17.29 \%\end{array}$ \\
\hline $\begin{array}{c}15 \\
\text { (26 y/o Male) }\end{array}$ & $\begin{array}{l}\text { Push-ups: } 79.5 \\
\text { Dips: } 70 \\
\text { Squats: } 81 \\
\text { Lunges: } 85.5\end{array}$ & $\begin{array}{l}\text { Push-ups: } 69 \\
\text { Dips: } 72.5 \\
\text { Squats: } 94.5 \\
\text { Lunges: } 96.5\end{array}$ & $\begin{array}{l}\text { Push-ups: } \downarrow 13.20 \% \\
\text { Dips: } \uparrow 3.57 \% \\
\text { Squats: } \uparrow 16.66 \% \\
\text { Lunges: } \uparrow 12.86 \%\end{array}$ & $\begin{array}{c}\text { OPUS increased } \\
\text { overall athletic } \\
\text { performance } 4.97 \%\end{array}$ \\
\hline
\end{tabular}


Table 2. Results from Placebo group including overall percentage change from baseline and percent changes for each particular exercise

\begin{tabular}{|c|c|c|c|c|}
\hline Participant \# & $\begin{array}{l}\text { Baseline } \\
\text { averages }\end{array}$ & $\begin{array}{l}\text { Placebo } \\
\text { averages }\end{array}$ & $\%$ Change & Conclusion \\
\hline $\begin{array}{c}6 \\
(28 \mathrm{y} / \mathrm{o} \text { Male) }\end{array}$ & $\begin{array}{l}\text { Push-ups: } 67 \\
\text { Dips: } 73.5 \\
\text { Squats: } 91.5 \\
\text { Lunges: } 93\end{array}$ & $\begin{array}{l}\text { Push-ups: } 69 \\
\text { Dips: } 74 \\
\text { Squats: } 88.5 \\
\text { Lunges: } 90.5\end{array}$ & $\begin{array}{l}\text { Push-ups: } \uparrow 2.98 \% \\
\text { Dips: } \uparrow 0.68 \% \\
\text { Squats: } \downarrow 3.27 \% \\
\text { Lunges: } \downarrow 2.68 \%\end{array}$ & $\begin{array}{c}\text { Placebo } \\
\text { decreased } \\
\text { overall athletic } \\
\text { performance } \\
1.14 \%\end{array}$ \\
\hline $\begin{array}{c}7 \\
(25 \mathrm{y} / \mathrm{o} \text { Male) }\end{array}$ & $\begin{array}{l}\text { Push-ups: } 37 \\
\text { Dips: } 53 \\
\text { Squats: } 68.5 \\
\text { Lunges: } 87\end{array}$ & $\begin{array}{l}\text { Push-ups: } 35.5 \\
\text { Dips: } 54 \\
\text { Squats: } 78 \\
\text { Lunges:78 }\end{array}$ & $\begin{array}{l}\text { Push-ups: } \downarrow 4.05 \% \\
\text { Dips: } \uparrow 1.88 \% \\
\text { Squats: } \uparrow 13.86 \% \\
\text { Lunges: } \downarrow 10.34 \%\end{array}$ & $\begin{array}{c}\text { Placebo } \\
\text { increased overall } \\
\text { athletic } \\
\text { performance } \\
1.35 \%\end{array}$ \\
\hline $\begin{array}{c}8 \\
(24 \mathrm{y} / \mathrm{o} \text { Female) }\end{array}$ & $\begin{array}{l}\text { Push-ups: } 65.5 \\
\text { Dips: } 72 \\
\text { Squats: } 88.5 \\
\text { Lunges: } 63.5\end{array}$ & $\begin{array}{l}\text { Push-ups: } 74.5 \\
\text { Dips: } 87 \\
\text { Squats: } 100.5 \\
\text { Lunges: } 59\end{array}$ & $\begin{array}{l}\text { Push-ups: } \uparrow 13.74 \% \\
\text { Dips: } \uparrow 20.83 \% \\
\text { Squats: } \uparrow 13.55 \% \\
\text { Lunges: } \downarrow 7.08 \%\end{array}$ & $\begin{array}{c}\text { Placebo } \\
\text { increased overall } \\
\text { athletic } \\
\text { performance } \\
10.26 \%\end{array}$ \\
\hline $\begin{array}{c}9 \\
\text { (21 y/o Female) }\end{array}$ & $\begin{array}{l}\text { Push-ups: } 56.5 \\
\text { Dips: } 77.5 \\
\text { Squats: } 93.5 \\
\text { Lunges: } 73\end{array}$ & $\begin{array}{l}\text { Push-ups: } 54.5 \\
\text { Dips: } 77 \\
\text { Squats: } 92.5 \\
\text { Lunges: } 73.5\end{array}$ & $\begin{array}{l}\text { Push-ups: } \downarrow 3.53 \% \\
\text { Dips: } \downarrow 0.64 \% \\
\text { Squats: } \downarrow 1.06 \% \\
\text { Lunges: } \uparrow 0.68 \%\end{array}$ & $\begin{array}{c}\begin{array}{c}\text { Placebo } \\
\text { decreased }\end{array} \\
\text { overall athletic } \\
\text { performance } \\
1.13 \%\end{array}$ \\
\hline $\begin{array}{c}10 \\
(23 \mathrm{y} / \mathrm{o} \text { Female) }\end{array}$ & $\begin{array}{l}\text { Push-ups: } 36.5 \\
\text { Dips: } 98 \\
\text { Squats: } 232.5 \\
\text { Lunges: } 94\end{array}$ & $\begin{array}{l}\text { Push-ups: } 30.5 \\
\text { Dips: } 88.5 \\
\text { Squats: } 178.5 \\
\text { Lunges: } 93.5\end{array}$ & $\begin{array}{l}\text { Push-ups: } \downarrow 16.43 \% \\
\text { Dips: } \downarrow 9.69 \% \\
\text { Squats: } \downarrow 23.22 \% \\
\text { Lunges: } \downarrow 0.53 \%\end{array}$ & $\begin{array}{c}\begin{array}{c}\text { Placebo } \\
\text { decreased }\end{array} \\
\text { overall athletic } \\
\text { performance } \\
12.46 \%\end{array}$ \\
\hline $\begin{array}{c}16 \\
(22 \mathrm{y} / \mathrm{o} \text { Male) }\end{array}$ & $\begin{array}{l}\text { Push-ups: } 82.5 \\
\text { Dips: } 82.5 \\
\text { Squats: } 89.5 \\
\text { Lunges: } 59\end{array}$ & $\begin{array}{l}\text { Push-ups: } 81.5 \\
\text { Dips: } 102.5 \\
\text { Squats: } 56 \\
\text { Lunges: } 54\end{array}$ & $\begin{array}{l}\text { Push-ups: } \downarrow 1.21 \% \\
\text { Dips: } \uparrow 24.24 \% \\
\text { Squats: } \downarrow 37.43 \% \\
\text { Lunges: } \downarrow 8.47 \%\end{array}$ & $\begin{array}{c}\begin{array}{c}\text { Placebo } \\
\text { decreased }\end{array} \\
\text { overall athletic } \\
\text { performance } \\
5.71 \%\end{array}$ \\
\hline
\end{tabular}




\begin{tabular}{|c|c|c|c|c|}
\hline $\begin{array}{c}17 \\
(27 \mathrm{y} / \mathrm{o} \text { Male })\end{array}$ & $\begin{array}{l}\text { Push-ups: } 41.5 \\
\text { Dips: } 36.5 \\
\text { Squats: } 92.5 \\
\text { Lunges: } 80\end{array}$ & $\begin{array}{l}\text { Push-ups: } 46.5 \\
\text { Dips: } 39 \\
\text { Squats: } 94.5 \\
\text { Lunges: } 78.5\end{array}$ & $\begin{array}{l}\text { Push-ups: } \uparrow 12.04 \% \\
\text { Dips: } \uparrow 6.84 \% \\
\text { Squats: } \uparrow 2.16 \% \\
\text { Lunges: } \downarrow 1.87 \%\end{array}$ & $\begin{array}{c}\text { Placebo } \\
\text { increased overall } \\
\begin{array}{c}\text { athletic } \\
\text { performance }\end{array} \\
4.79 \%\end{array}$ \\
\hline $\begin{array}{c}18 \\
\text { (22 y/o Female) }\end{array}$ & $\begin{array}{l}\text { Push-ups: } 64.5 \\
\text { Dips: } 53.5 \\
\text { Squats: } 132.5 \\
\text { Lunges: } 104\end{array}$ & $\begin{array}{l}\text { Push-ups: } 61.5 \\
\text { Dips: } 54.5 \\
\text { Squats: } 134 \\
\text { Lunges: } 102\end{array}$ & $\begin{array}{l}\text { Push-ups: } \downarrow 4.65 \% \\
\text { Dips: } \uparrow 1.86 \% \\
\text { Squats: } \uparrow 1.13 \% \\
\text { Lunges: } \downarrow 1.92 \%\end{array}$ & $\begin{array}{c}\text { Placebo } \\
\text { decreased } \\
\text { overall athletic } \\
\text { performance } \\
1.79 \%\end{array}$ \\
\hline $\begin{array}{c}19 \\
\text { (24 y/o Female) }\end{array}$ & $\begin{array}{l}\text { Push-ups: } 14 \\
\text { Dips: } 22 \\
\text { Squats: } 113.5 \\
\text { Lunges: } 66\end{array}$ & $\begin{array}{l}\text { Push-ups: } 17.5 \\
\text { Dips: } 17.5 \\
\text { Squats: } 112.5 \\
\text { Lunges: } 73.5\end{array}$ & $\begin{array}{l}\text { Push-ups: } \uparrow 25 \% \\
\text { Dips: } \downarrow 20.45 \% \\
\text { Squats: } \downarrow 0.88 \% \\
\text { Lunges: } \uparrow 11.36 \%\end{array}$ & $\begin{array}{c}\text { Placebo } \\
\text { increased overall } \\
\text { athletic } \\
\text { performance } \\
3.75 \%\end{array}$ \\
\hline $\begin{array}{c}20 \\
(20 \mathrm{y} / \mathrm{o} \text { Female) }\end{array}$ & $\begin{array}{l}\text { Push-ups: } 60 \\
\text { Dips: } 48 \\
\text { Squats: } 121 \\
\text { Lunges: } 112\end{array}$ & $\begin{array}{l}\text { Push-ups: } 56.5 \\
\text { Dips: } 58.5 \\
\text { Squats: } 120.5 \\
\text { Lunges: } 110\end{array}$ & $\begin{array}{l}\text { Push-ups: } \downarrow 5.83 \% \\
\text { Dips: } \uparrow 21.78 \% \\
\text { Squats: } \downarrow 0.43 \% \\
\text { Lunges: } \downarrow 1.78 \%\end{array}$ & $\begin{array}{c}\text { Placebo } \\
\text { increased overall } \\
\text { athletic } \\
\text { performance } \\
3.45 \%\end{array}$ \\
\hline
\end{tabular}




\section{References}

1) Artioli G, Gualano B, Smith A, et al. Role of B-Alanine Supplementation on Muscle Carnosine and Exercise Performance. Journal of the American College of Sports Medicine. 2010; 42(6): 1162-1173.

2)Barrett K.E., Barman S.M., Boitano S., Brooks H.L. (2012). Chapter 26. Digestion, Absorption, \& Nutritional Principles. In K.E. Barrett, S.M. Barman, S. Boitano, H.L. Brooks (Eds), Ganong's Review of Medical Physiology, 24e.

3) Barrows K. (2013). Chapter e5. Complementary \& Alternative Medicine. In M.A. Papadakis, S.J. McPhee, M.W. Rabow (Eds), CURRENT Medical Diagnosis \& Treatment 2013.

4) Benardot D. Training with supplements: determining which ergogenic aids can improve performance and performance nutrition plans: combined power and endurance sports, in Nutrition for serious athletes; an advanced guide to foods, fluids and supplements for training and performance. In: Benardot D, editor. Nutrition for serious athletes. Chicago: Human Kinetics; 2000. pp. 123-37.

5) Braen G.R., Joshi P. (2011). Chapter 199. Vitamins and Herbals. In J.E. Tintinalli, J.S. Stapczynski, D.M. Cline, O.J. Ma, R.K. Cydulka, G.D. Meckler (Eds), Tintinalli's Emergency Medicine: A Comprehensive Study Guide, 7e.

6) Briggs J.P. (2012). Chapter e2. Complementary, Alternative, and Integrative Medicine. In D.L. Longo, A.S. Fauci, D.L. Kasper, S.L. Hauser, J.L. Jameson, J. Loscalzo (Eds), Harrison's Principles of Internal Medicine, 18e.

7) Bryant PJ, Norris KP, McQueen CE, Poole EA. Chapter 5. Literature Evaluation II: Beyond the Basics. In: Kier KL, Malone PM, Stanovich JE, eds. Drug Information: A Guide for Pharmacists. 4th ed. New York: McGraw-Hill; 2012.

8) Burns RD, Schiller MR, Merrick MA, Wolf KN. Intercollegiate student athlete use of nutritional supplements and the role of athletic trainers and dietitians in nutrition counseling. J Am Diet Assoc. 2004;104(2):246-9.

9) Dennehy C.E., Tsourounis C. (2012). Chapter 64. Dietary Supplements \& Herbal Medications. In B.G. Katzung, S.B. Masters, A.J. Trevor (Eds), Basic \& Clinical Pharmacology, $12 \mathrm{e}$.

10) Duque G., Troen B.R. (2009). Chapter 117. Osteoporosis. In J.B. Halter, J.G. Ouslander, M.E. Tinetti, S. Studenski, K.P. High, S. Asthana (Eds), Hazzard's Geriatric Medicine and Gerontology, 6e.

11) Dwyer J. (2012). Chapter 73. Nutrient Requirements and Dietary Assessment. In D.L. Longo, A.S. Fauci, D.L. Kasper, S.L. Hauser, J.L. Jameson, J. Loscalzo (Eds), Harrison's Principles of Internal Medicine, 18e.

12) Froiland K, Koszewski W, Hingst J, Kopecky L. Nutritional supplement use among college athletes and their sources of information. Int $\mathrm{J}$ Sport Nutr Exerc Metab.
2004;14(1):104-20.

13) Ganio M, Klau J, Casa D, et al. Effect of Caffeine on Sport-Specific Endurance Performance: A Systematic Review. Journal of Strength and Conditioning Research. 2009; 23(1): 315-324.

14) Gomella L.G., Haist S.A. (2007). Chapter 11. Nutritional Assessment, Therapeutic Diets, and Infant Feeding. In L.G. Gomella, S.A. Haist (Eds), Clinician's Pocket Reference: The Scut Monkey, 11e.

15) Haller C.A., Ko R. (2012). Chapter 79. Herbal and Alternative Products. In K.R. Olson (Ed), Poisoning \& Drug Overdose, 6e.

16) Kaul P. (2012). Chapter 5. Adolescent Substance Abuse. In W.W. Hay, Jr., M.J. Levin, R.R. Deterding, J.J. Ross, J.M. Sondheimer (Eds), CURRENT Diagnosis \& Treatment: Pediatrics, 21e.

17) Kern B, Robinson TL, Manninen AH. Effects of betaalanine supplementation on exercise performance during a competitive wrestling season: an 8-week open label pilot study. Journal of the International Society of Sports Nutrition. 2008;5(Suppl 1):P2.

18) Krucoff M.W., Costello R.B., Mark D., Vogel J.H. (2011). Chapter 115. Complementary and Alternative Medical Therapy in Cardiovascular Care. In V. Fuster, R.A. Walsh, R.A. Harrington (Eds), Hurst's The Heart, 13e.

19) Molinero O, Marquez S. Use of nutritional supplements in sports: risks, knowledge, and behavioural-related factors. Nutrición Hospitalaria. 2009; 24(2): 128-34.

20) Morgan, Jr. G.E., Mikhail M.S., Murray M.J. (2006). Chapter 49. Critical Care. In G.E. Morgan, Jr., M.S. Mikhail, M.J. Murray (Eds), Clinical Anesthesiology, 4e.

21) Murray R.K. (2011). Chapter 1. Biochemistry \& Medicine. In D.A. Bender, K.M. Botham, P.A. Weil, P.J. Kennelly, R.K. Murray, V.W. Rodwell (Eds), Harper's Illustrated Biochemistry, 29e.

22) Natural Medicines Comprehensive Database. Stockton, CA: Therapeutic Research Faculty; 2010. http://naturaldatabase.therapeuticresearch.com/. Accessed March 25, 2013.

23) Olson K.R. (2013). Chapter 38. Poisoning. In M.A. Papadakis, S.J. McPhee, M.W. Rabow (Eds), CURRENT Medical Diagnosis \& Treatment 2013.

24) Rahnama N, Gaeini AA, Kazemi F. The effectiveness of two energy drinks on selected indices of maximal cardiorespiratory fitness and blood lactate levels in male athletes. J Res Med Sci. 2010;15(3):127-32.

25) Richmond, Scott R, Godard, et al. The Effects of Varied Rest Periods Between Sets to Failure Using the Bench Press in Recretionally Trained Men. Journal of Strength \& Conditioning Research. 2004; 18(4): 689-983. 
26) Sale C, Saunders B, Harris R. Effect of beta-alanine supplementation on muscle carnosine concntrations and excercise performance. Amino Acids. 2010; 39(2): 321-333.

27) Shields KM, Blythe E. Chapter 3. Drug Information Resources. In: Kier KL, Malone PM, Stanovich JE, eds. Drug Information: A Guide for Pharmacists. 4th ed. New York: McGraw-Hill; 2012.

28) Sobal J, Marquart LF. Vitamin/mineral supplement use among athletes: a review of the literature. Int J Sport Nutr. 1994;4(4):320-34.

29) Stephens M.B., Burnett D. (2011). Chapter 10. Physical Activity in Adolescents. In J.E. South-Paul, S.C. Matheny, E.L. Lewis (Eds), CURRENT Diagnosis \& Treatment in Family Medicine, 3e.

30) Stout JR, Moon JR, Tobkin SE, Lockwood CM, Smith AE, Graef JL, et al. Pre-workout consumption of Celsius ${ }^{\circledR}$ enhances the benefits of chronic exercise on body composition and cardiorespiratory fitness. Journal of the
International Society of Sports Nutrition. 2008;5(Suppl 1):P8.

31) Tian HH, Ong WS, Tan CL. Nutritional supplement use among university athletes in Singapore. Singapore Medical Journal. 2009; 50(2): 165-72.

32) Trevor AJ, Katzung BG, Masters SB. Chapter 60. Dietary Supplements \& Herbal Medications. In: Trevor AJ, Katzung BG, Masters SB, eds. Pharmacology: Examination \& Board Review. 9th ed. New York: McGraw-Hill; 2010.

33) Zadeh K.K., Kopple J.D. (2009). Chapter 21. Chronic Renal Failure \& the Uremic Syndrome: Nutritional Issues. In E.V. Lerma, J.S. Berns, A.R. Nissenson (Eds), CURRENT Diagnosis \& Treatment: Nephrology \& Hypertension.

34) Ziegenfuss $T$, Landis $J$, Hofheins J. Effect of a supplement containing primarily beta alanine, arginine, creatine malate, and glycerol monostearate on exerciseinduced changes in lean mass of the arms. Journal of the International Society of Sports Nutrition. 2008;5(Suppl 1):P16. 